\title{
Complex Dielectric Function and Optical Properties for the Perovskite BCZT via VEELS-STEM.
}

G. Herrera-Perez ${ }^{1}$, Carlos Ornelas-Gutiérrez ${ }^{2}$, Sergio Marungo-Ramirez ${ }^{3}$, Armando Reyes-Rojas ${ }^{4}$ and Luis Fuentes-Cobas 4

${ }^{1}$ Centro de Investigación en Materiales Avanzados, Chihuahua, Chihuahua, Mexico, ${ }^{2}$ Centro de Investigación en Materiales Avanzados, ${ }^{3}$ Universidad Tecnologica de Chihuahua campus Sur, United States, ${ }^{4}$ Centro de Investigacion en Materiales Avanzados, United States

Valence electron energy-loss spectroscopy scanning transmission electron microscopy (VEELS-STEM) is an alternative option to elucidate the electronic structure of materials in the region between 0 to $50 \mathrm{eV}$ [1]. The analysis of this region using the Kramers-Kronig $(\mathrm{K}-\mathrm{K})$ relations allows determining the complex dielectric function, $\varepsilon^{*}=\varepsilon 1+\mathrm{i} \varepsilon 2$ [1]. The monitoring of optical properties such as absorption coefficient $(\mu)$, refractive index $(\mathrm{n})$ and the extinction coefficient $(\mathrm{k})$ of materials is determined via the dielectric function. There are no reports in the literature that exhibits the $\mathrm{K}-\mathrm{K}$ analysis of VEELS for the perovskite $\mathrm{Ba} 0.8 \mathrm{Ca} 0.2 \mathrm{Ti0.9Zr0.1O3}$ (BCZT) compound. This compound is a good lead-free ferro-piezoelectric material that exhibits a tetragonal phase [2]. The motivation of this work is to present the dielectric function and the optical properties for perovskite BCZT via VEELS-STEM analysis using the K-K relations.

BCZT was prepared by the modified Pechini method, the experimental details were reported elsewhere [3]. EELS spectra were obtained with an electron energy loss spectrometer (EELS GAT-777 STEMPack) attached to a JEM-2200FS (200 kV), which offers an energy resolution of $1.0 \mathrm{eV}$. The electron probe size was below $1 \mathrm{~nm}$. The spectra were acquired using a dispersion of $0.05 \mathrm{eV} /$ channel to record spectra up to $1000 \mathrm{eV}$. The convergence semi-angle was $\alpha=9.0 \mathrm{mrad}$ for a $2.5 \mathrm{~mm}$ spectrometer entrance aperture and $40 \mathrm{~mm}$ camera length, and the corresponding collection semi-angle was $\beta=17.3 \mathrm{mrad}[2]$. The deconvolution of the zero-loss peak (ZLP) from the VEELS region, the Fourier-log method to remove plural scattering and $\mathrm{K}-\mathrm{K}$ analysis were carried out using the Gatan Suite software [4].

Figure 1a shows the VEELS-STEM region where the ZLP is identified and elemental analysis is performed for the perovskite BCZT. Panel $b$ shows the ZLP that was extracted from the VEELS region considering in the fit the extrapolation of a logarithmic function [4]. The dominant broad peak located at $27.1 \mathrm{eV}$ is attributed to the bulk plasmon and the peak located at $13.6 \mathrm{eV}$ is related to the surface plasmon. The inelastic contribution spectrum was corrected for multiple scattering events using the Fourier-log method [4] obtaining the single scattering distribution (SSD). A polynomial fit up to the $\mathrm{x}$-axis suggests bandgap energy, Eg of $3.2 \mathrm{eV}$ (panel c) which agrees with the value reported in the literature. Figure 2a exhibits the complex dielectric function, where $\varepsilon 1$ and $\varepsilon 2$ are, respectively, the real and imaginary parts of $\varepsilon$ [1]; and the imaginary part of $\{\varepsilon-1\}$ also known as the energy loss function (ELF). These curves were obtained through the $\mathrm{K}-\mathrm{K}$ analysis [4]. Refractive index (n) and $\mathrm{k}$ behavior in the range of $0-50 \mathrm{eV}$ are displayed in panel b. Optical absorption coefficient as calculated from $\varepsilon 1, \varepsilon 2$ (panel c). Panel d shows an optical region expanded.

VEELS-STEM technique combined with $\mathrm{K}-\mathrm{K}$ analysis is a very useful technique to monitor (a) the elemental composition and locate the plasmon contributions in the valence region; (b) the complex dielectric function, and (c) to determine the bandgap energy, refractive index, extinction and absorption coefficient in particular for ferro-piezoelectric materials such as BCZT. 
G. H-P thanks to CONACyT-SEP Basic Research Project No. 253605 and catedras-CONACYT program No. 580.
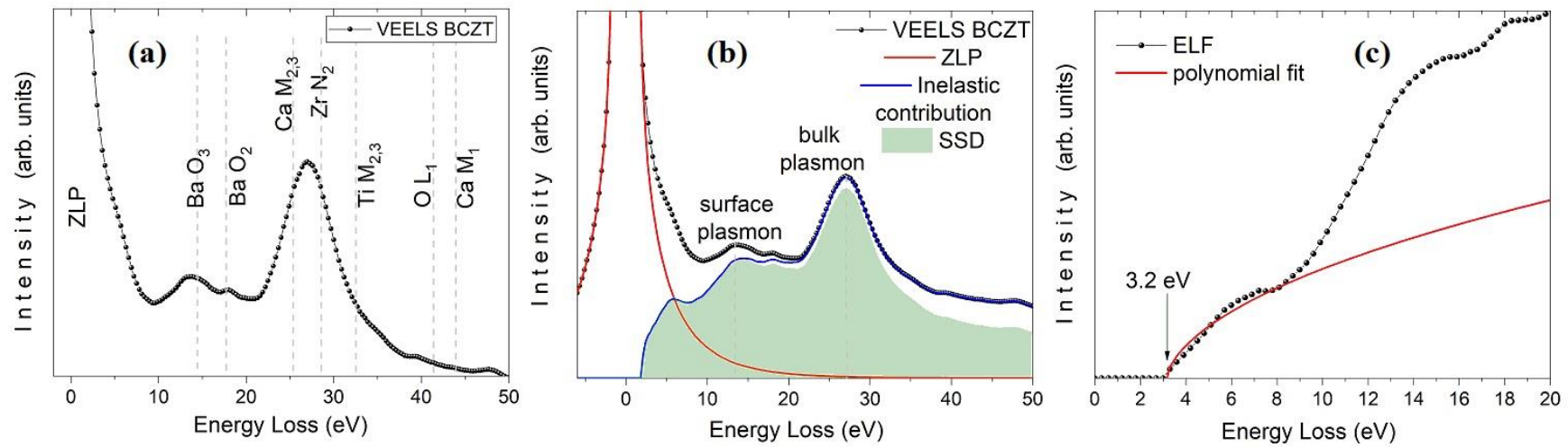

Figure 1. (a) Elemental analysis in the VEELS region. (b) ZLP deconvolution, inelastic contribution, bulk plasmon and SSD. (c) Bandgap determination using a polynomial fit in the ELF for perovskite BCZT.
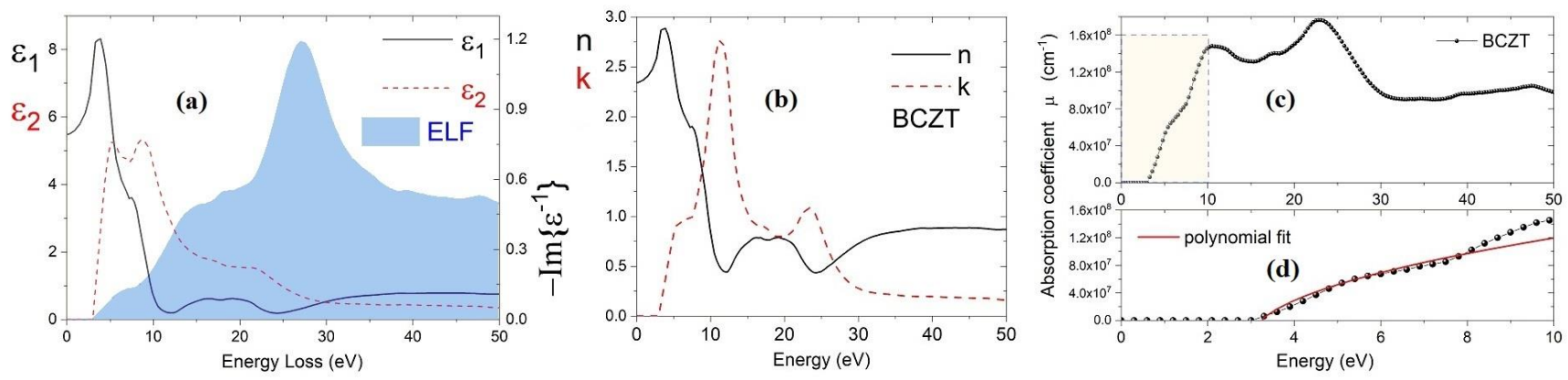

Figure 2. (a) Dielectric function and ELF. (b) Refractive index, $n$ and extinction coefficient, k. (c) The optical absorption coefficient, $\mu$. (d) Optical region expanded for ferro-piezoelectric BCZT.

\section{References}

[1] P. Y. Yu and M. Cardona. Fundamentals of semiconductors, Springer, Berlin 1999.

[2] G. Herrera-Pérez et al, J. Appl. Phys. 128 (2020), 064106.

[3] G. Herrera-Pérez et al, J. Appl. Phys. 120 (2016), 094303.

[4] Digital Micrograph EELS Analysis User's Guide. Gatan, Inc. (2003) USA. 